\title{
Asymmetric Cryptography with a Hidden Monomial
}

\author{
and a candidate algorithm for $\simeq 64$ bits asymmetric signatures
}

\author{
Jacques Patarin \\ CP8 TRANSAC , 68 route de Versailles - BP 45 \\ 78431 Louveciennes Cedex - France \\ e-mail : J.Patarin@frlv.bull.fr
}

\begin{abstract}
In [1] T. Matsumoto and H. Imai have presented a very efficicnt "candidate" algorithm, called $C^{*}$, for asymmetric cryptography. This algorithm was broken in [2]. Then in [3], I have suggested two algorithms, HFE and IP, to repair $C^{*}$. However the secret key computations of HFE and IP are not as efficient as in the original algorithm $C^{*}$. Is it possible to repair $C^{*}$ with the same kind of very easy secret key computations? This question is the subject of this paper. Unfortunately, we will see that for all the "easy" transformations of $C^{*}$ the answer is no. However one of the new ideas of this paper will enable us to suggest a candidate algorithm for assymetric signatures of length only 64 bits. An extended version of this paper can be obtained from the author.
\end{abstract}

\section{Introduction}

In [1] 'I'. Matsumoto and $\mathrm{H}$. Imai have presented a very efficient algorithm $C^{*}$ for asymmetric cryptography (authentications, signatures or encryptions) with public multivariate quadratic polynomials. This algorithm was based on the idca of "hiding" a monomial equation $b=f(a)=a^{1+2^{\theta}}$ by two affine permutations $s$ and $t$. In [2], I have shown that this original algorithm was insecure. Then in [3], I have suggested two new algorithms IIFE and IP in order to repair $C^{*}$. HFE use more complex hidden functions $f$ (functions $f$ with more than one monomial and sometimes also more than one variable $a$ ) but the computation of $f^{-1}$ with the secret key is (of course still feasable but is) more difficult than in $C^{*}$. IP is a very different algorithm. It looks like the famous Graph Isomorphisms algorithm.

Is is possible to repair $C^{*}$ and keeping the same kind of easy secret key computations? For example with multivariate polynomials of total degree $3 \mathrm{or}$ 4 in the public form (instead of two) if necessary? This question is the subject of this paper. 
First we will describe two new asymmetric "candidate" algorithms: Dragon and MIIP-3. These algorithms are very efficient. Then we will see that some easier algorithms are insecure. Then we will extend our attacks to see that Dragon with one hidden monomial and MIIP-3 are also insecure.

So it seems that there is not an easy way to "hide" a monomial in order to avoid polynomial attacks ... Nevertheless at the end of this paper, we will show that the idea of "Dragon" Algorithms (however with more than one monomial) gives us a candidate algorithm for extremely short asymmetric signatures. Moreover another family of algorithms (not described here) is still under investigation.

\section{PART 1: Description of the hidden monomial schemes}

\section{2 "Dragon": a new family of algorithms for asym- metric cryptography}

\section{The public polynomials of the "Dragon" family}

The first family of algorithms that we will describe is called "Dragon". Before going into details, let us start by showing the differences between the public polynomials of a scheme like Matsumoto-Imai $C^{*}$ scheme of [1] and the Dragon schemes.

- In Matsumoto-Imai $C^{*}$ scheme (or in my HFE scheme of [3]), the public equations are $n$ multivariate polynomials $P_{1}, \ldots, P_{n}$ over a finite field $K$, ( $n$ integer), and these polynomials give $y_{1}, \ldots, y_{n}$ as functions of $x_{1}, \ldots, x_{n}$ like this:

$$
\left\{\begin{aligned}
y_{1} & =P_{1}\left(x_{1}, \ldots, x_{n}\right) \\
y_{2} & =P_{2}\left(x_{1}, \ldots, x_{n}\right) \\
& \vdots \\
y_{n} & =P_{n}\left(x_{1}, \ldots, x_{n}\right)
\end{aligned}\right.
$$

where in encryption $\left(x_{1}, \ldots, x_{n}\right)$ is the cleartext and $\left(y_{1}, \ldots, y_{n}\right)$ the ciphertext (in signature $\left(x_{1}, \ldots, x_{n}\right)$ is the signature and $\left(y_{1}, \ldots, y_{n}\right)$ the message to sign or a public transformation of the message to sign). Moreover in $C^{*}$ Algorithm the polynomials $P_{1}, \ldots, P_{n}$ have total degree 2.

- In the Dragon algorithms that we will describe the public equations are $\lambda$ multivariate polynomials over a field $K$ (or a ring) like this:

$$
\left\{\begin{aligned}
P_{1}\left(x_{1}, \ldots, x_{n}, y_{1}, \ldots, y_{m}\right) & =0 \\
P_{2}\left(x_{1}, \ldots, x_{n}, y_{1}, \ldots, y_{m}\right) & =0 \\
& \vdots \\
P_{\lambda}\left(x_{1}, \ldots, x_{n}, y_{1}, \ldots, y_{m}\right) & =0
\end{aligned}\right.
$$

where $P_{1}, P_{2}, \ldots, P_{\lambda}$ are polynomials of $K^{n} \times K^{m} \rightarrow K$ of small total degree (for example 2,3 or 4 ). 
As before in encryption $\left(x_{1}, \ldots, x_{n}\right)$ is the cleartext and $\left(y_{1}, \ldots, y_{m}\right)$ the ciphertext (in signature $\left(x_{1}, \ldots, x_{n}\right)$ is the signature and $\left(y_{1}, \ldots, y_{m}\right)$ the message to sign or a public transformation of the message to sign).

So the big difference in the public equations between the Dragon algorithms and Matsumoto-Imai algorithms is that we have "mixed" the variables $x_{i}$ and $y_{i}$.

\section{First example of Dragon in encryption}

Here $\lambda=m=n$ and $K$ is a small finite field. Let $q=|K|$ be the number of elements of $K$. For example $K=F_{2}=C F(2)$ the finite field with two elements.

$\left(x_{1}, \ldots, x_{n}\right) \in K^{n}$ is the cleartext. $\left(y_{1}, \ldots, y_{n}\right) \in K^{n}$ is the ciphertext. If we have the secrets then we can obtain $\left(y_{1}, \ldots, y_{n}\right)$ from $\left(x_{1}, \ldots, x_{n}\right)$ like this (we will see below another way to compute $\left(y_{1}, \ldots, y_{n}\right)$ from $\left(x_{1}, \ldots, x_{n}\right)$ without any secrets):

1. $x=\left(x_{1}, \ldots, x_{n}\right)$ is first transformed with an affine secret permutation $s$, so we obtain $s(x)=a=\left(a_{1}, \ldots, a_{n}\right)$.

2. Then $a$ is transformed in $b$ such that

$$
a^{q^{\natural}+q^{\varphi}} \cdot M(b)=a^{q^{\zeta}+q^{\xi}} \cdot N(b)
$$

where $\theta, \varphi, \zeta, \xi$ are secret or public intcgers such that $h=q^{\theta}+q^{\varphi}-q^{\zeta}-q^{\xi}$ is coprime with $q^{n}-1, q=|K|$, where the exponentiations are done in a representation of the field $\mathbb{F}_{q^{n}}$, and where $M$ and $N$ are two affine functions( we will comment the choice of $M$ and $N$ below).

How do we compute $b$ from $a$ ? (We will now give a general way to compute $b$ from $a$ but we will see below that there are sometimes some easicr ways). If we write the equation (1) in the components $\left(a_{1}, \ldots, a_{n}\right)$ and $\left(b_{1}, \ldots, b_{n}\right)$ of $a$ and $b$ (i.e. in a basis of $F_{q^{n}}$ ), we will obtain $n$ equations like this:

$$
\sum \gamma_{i j k} a_{i} a_{j} b_{k}+\sum \mu_{i . j} a_{i} a_{j}=0
$$

where $\gamma_{i j}$ and $\mu_{i j}$ are some coefficients of $K$.

The reason for this is that $x \mapsto x^{q^{\theta}}$ and $x \mapsto x^{q^{\varphi}}$ are linear functions of $F_{q^{n}}$, so $x \mapsto x^{q^{\theta}+q^{\varphi}}$ in a basis and $x \mapsto x^{q^{\zeta}+q^{\xi}}$ are given by quadratic polynomials.

Now when $\left(a_{1}, \ldots, a_{n}\right)$ is given the $n$ equations (2) give $n$ equations of degree 1 in the values $b_{i}$. So by Gaussian reduction it is then easy (on a computer) to find all the solutions of these equations. We will assume that at least one solution $b$ is found such that $M(b) \neq 0$ or $N(b) \neq 0$. (we will comment this point at the end of this paragraph). If more than one such $b$ is found, we randomly chose one of the solutions for $b$.

3 . Finally $b=\left(b_{1}, \ldots, b_{n}\right)$ is transformed with another affine secret permutation $l$, so we obtain $t(b)=y=\left(y_{1}, \ldots, y_{n}\right)$. 
Remark All these operations are invertibles, so it is possible to compute $\left(x_{1}, \ldots, x_{n}\right)$ from $\left(y_{1}, \ldots, y_{n}\right)$ if the secrets $s, t, \theta, \varphi, \zeta, \xi$ and the representation of the field $F_{q^{n}}$ are known. For example if $M(b) \neq 0$ then $a$ will be found from $b$ by:

$a=(N(b) / M(b))^{h^{\prime}}$ wherc $h^{\prime}$ is the inverse of $h=q^{\theta}+q^{\varphi}-q^{\zeta}-q^{\xi}$ modulo $q^{n}-1$.

\section{Public computation of $\left(y_{1}, \ldots, y_{n}\right)$ from $\left(x_{1}, \ldots, x_{n}\right)$}

The $n$ equations (2) will be transformed in a system of $n$ equations like this:

$$
\sum \alpha_{i j k} x_{i} x_{j} y_{k}+\sum \beta_{i j} x_{i} x_{j}+\sum \nu_{i j} x_{i} y_{i}+\sum \varepsilon_{i} x_{i}+\sum \zeta_{i} y_{i}+\delta_{0}=0
$$

i.e. $n$ equations $P_{i}\left(x_{1}, \ldots, x_{n}, y_{1}, \ldots, y_{n}\right)=0, i=1, \ldots, n$, where $P_{i}$ is a polynomial of $K^{2 n} \rightarrow K$, of total degree three. These $n$ equations (3) will be public. They are the public key.

The computation of the $n$ equations (3) from the $n$ equations (2) is done in two steps: first we replace the $b_{i}$ by their affine expression in $y_{j}$ and the $a_{i}$ by their affine expression in $x_{i}$ (Step 1). 'Then a linear and bijective transformation $u$ is done on these equations (Step 2).

Note 1. This Step 2 transformation $u$ is secret, or is done in a way to have equations (3) with a conventional presentation (for cxample the equation number $k,(1 \leq k \leq n)$ will have a term in $x_{1} x_{2} y_{k}$ and no terms in $x_{1} x_{2} y_{j}, j \neq k$ : this gives a conventional presentation obtained by Caussian reductions).

Note 2. We will see in paragraph 4 that the public key length can be moderate despite the fact that the public polynomials are of total degree three. With these public equations (3) anybody will be able to encrypt a message, i.e. to compute $\left(y_{1}, \ldots, y_{n}\right)$ from $\left(x_{1}, \ldots, x_{n}\right)$ without any secret (this is always feasable if there is a value $b$ such that (1) is satisfied).

The reason for this is that when $\left(x_{1}, \ldots, x_{n}\right)$ are given, the $n$ equations $(3)$ give $n$ equations of degree 1 in the values $y_{i}$. So by Gaussian reduction it is then easy to find all the solutions of these equations.

Remark. What is unusual with this Dragon Algorithms is that although anybody can compute $\left(y_{1}, \ldots, y_{n}\right)$ from $\left(x_{1}, \ldots, x_{n}\right)$ nobody can express the $x_{i}$ variables as an effective polynomial in the $y_{j}$ variables (this polynomial exist but is too large to be explicit if the parameters are well chosen). What is also unusual is the fact that these "Dragon Algorithms" use in a the way the cryptanalysis algorithms of [2] (i.e. with Gaussian reduction) in order to design a new cryptosystems. 


\section{The first example of Dragon in signature}

It is casy to use this little Dragon Algorithms for asymmetric signatures. For example if $\left(y_{1}, \ldots, y_{n}\right)$ is the message to sign (or a public transformation of the message to sign), then $\left(x_{1}, \ldots, x_{n}\right)$ will be the signature. (The value $x$ corresponding to $a=0$ may be public in order to avoid this value to be a valid signature of any message).

\section{About the choice of $M$ and $N$}

There are different ways to choose $M$ and $N$.

Example 1 In this example, $M$ and $N$ are two secret random affine functions. In signature this Dragon Algorithm is very efficient, but in encryption we may have no solution in $b$ for equation (1).

However, the probability is high to find a solution $b$ (if $q$ is not too small). (See the extended version for more details). Moreover in the design of the scheme we can decide that a few bytes of the message $x$ have no information, and in the case we find no $y$ for a specific $x$, we can change these bytes and try again.

Example 2 In this example $M(b)=b$ and $N(b)=\mu b^{q^{\alpha}}+\nu b$ where $\alpha$ is an integer such that $q^{\alpha}-1$ is coprime with $q^{n}-1$ and where $\mu$ and $\nu$ are two elements of $F_{q^{n}}$ with $\mu \neq 0$ (but $\nu=0$ is possible). So the equation (1) is:

$$
a^{q^{\theta}+q^{\varphi}} \cdot b=a^{q^{\varsigma}+q^{\varepsilon}} \cdot\left(\mu b^{q^{\alpha}}+\nu b\right) .
$$

Now for each $a=0$ there is exactly only one $b \neq 0$ such that (4) is satisfied.

So this example 2 is an example of candidate trapdoor one way permutation! Moreover here the computation of b from a can be done by square and multiplicity (instead of Gaussian reductions).

Example 3 In this example $M(b)=b$ and $N(b)=\alpha b+1$, where $\alpha$ is a secret element of $F_{q^{n}}, \alpha \neq 0$. So the equation (Q) gives

$$
b=1 /\left(a^{q^{\theta}+q^{\varphi}-q^{\zeta}-i^{\xi}}-\alpha\right)
$$

So here again we have a candidate trapdoor one way permutation!

\section{The algorithm MIIP-3}

We will now see a second family of algorithms.

\section{Description of the algorithm}

As usual, lct $K$ be a finite field. Let $L_{n}$ be an extension of degree $n$ of $K$. Let $x$ and $y$ be two clements of $L_{n}$. In a basis $x$ is represented by $\left(x_{1}, x_{2}, \ldots, x_{n}\right)$ and $y$ by $\left(y_{1}, \ldots, y_{n}\right)$ where $\forall i, 1 \leq i \leq n, x_{i}$ and $y_{i}$ are elements of $K$. Let $s$ and $t$ be two secret affine functions of $K^{n} \rightarrow K^{n}$. The transformation from $x$ to $y$ can be obtained by these steps (if the secrets are known): 
Step 1 Compute $a=s(x)$.

Step 2 Compute (in $L_{n}$ ): $b=a^{1+q^{\theta}+q^{\varphi}}$, where $q=|K|$ and $\theta$ and $\varphi$ are two integers, $1 \leq \theta \leq \varphi$, such that $h=1+q^{\theta}+q^{\varphi}$ is coprime with $q^{n}-1$.

Step 3 Finally compute $y=t(b)$.

In a basis each component $y_{i}$ of $y$ can be written as a polynomial $P_{i}$ of total degree three in the $x_{j}$ valucs, $1 \leq j \leq n$.

These $n$ polynomials $P_{i}, 1 \leq i \leq n$, are made public. So, from these public polynomials, anybody can compute $y$ from $x$ (in encryption $y$ is the encryption of $x$, and in signature $x$ is the signature of $y$ ). Now if the secrets are known it is also easy to compute $x$ from $y$ : each step is easily invertible. But it "seems" that if the secrets are not known then $x$ can not be computed for $y$ (we will study this point in paragraph 9).

We call this algorithm MIIP-3: Matsumoto-Inai with Improved Parameters of degree 3. Compared with the original Matsumoto-Imai $C^{*}$ Algorithm [1] we have made three important changes:

1. There is only one branch (i.e. after Step 1, the value $a$ is not split in several branches as in [1]). The reason for this will be given in paragraph 5 .

2. The transformation $b=f(a)$ gives polynomials of degree three (and not two as in [1]). 'The reason for this is that the cryptanalysis of transformations $b=a^{1+q^{\theta}}$ was given in [2].

3. The field $K$ is not necessary of characteristic 2. (In [1] the field $K$ was of characteristic 2 in order to find some $\theta$ such that $1+q^{\theta}$ be coprime with $q^{n}-1$. If $q$ is odd this is not possible).

Remark It is very easy to find some bad values for $\theta$ and $\varphi$. For example if $q=2, \theta=1$, and $\varphi=2$, then

$$
b=a^{7}, \text { so } b \cdot a=a^{8}
$$

and from this equation (5) it is easy to sec that the scheme can be attacked exactly as the original $C^{*}$ scheme in [2]. However for almost all the choices of $\theta$ and $\varphi$ the attack of [2] does not work against MIIP-3.

\section{Equations (G1), (G2), (G3)}

Since $b=a^{1+q^{\theta}+q^{\varphi}}$, these three general equations $(G 1),(G 2),(G 3)$ are always satisfied:

$$
\begin{aligned}
& a^{1+q^{\varphi}} \cdot b^{q^{\theta}}=b \cdot a^{q^{\theta}\left(q^{\theta}+q^{\varphi}\right)} \\
& a^{1+q^{\theta}} \cdot b^{q^{\varphi}}=b \cdot a^{q^{\varphi}\left(q^{\varphi}+q^{\theta}\right)} \\
& b^{q^{\varphi}} \cdot a^{q^{\theta}+q^{2 \theta}}=b^{q^{\theta}} \cdot a^{q^{\varphi}+q^{2 \varphi}}
\end{aligned}
$$


Note. In a basis (G1), (G2), (G3) give $3 n$ cquations. Generally these $3 n$ equations are "formally" independent, i.e. they generate a vector space of dimension $3 n$. However if we give some explicit values for $b, b \neq 0$, then we will now prove that (G1), (G2), (G3) will give only $2 n$ independent equations.

Proof Iet $A=a^{1+q^{\theta}}, B=a^{1+q^{\varphi}}$ and $C=a^{q^{\theta}+q^{\varphi}}$. Then :

$$
\begin{aligned}
B \cdot b^{q^{\theta}} & =b \cdot C^{q^{\theta}} \\
A \cdot b^{q^{\psi}} & =b \cdot C^{q^{\psi}} \\
A^{q^{\theta}} \cdot b^{q^{\psi}} & =b^{q^{\psi}} \cdot B^{q^{\psi}}
\end{aligned}
$$

and let us assume that $b$ is known, and that $A, B$ and $C$ are unknown. Then from $(G 1),(G 2)$ and $(G 3)$ will we be able to find $A, B$, and $C$ ? No, because $(G 3)$ is just a consequence of $(G 1)$ and $(G 2)$ : from $\left(G^{*} 1\right)$ we have $A=b^{1-q^{\theta}} \cdot C^{q^{\theta}}$, and from $(G 2)$ we have $A=b^{1-q^{\varphi}} \cdot C^{q^{\varphi}}$. So $A^{q^{\theta}} \cdot b^{q^{\varphi}}=b^{q^{\theta}-q^{\theta+\varphi}+q^{\varphi}} \cdot C^{q^{\theta+\varphi}}=b^{q^{\theta}} \cdot B^{q^{\theta} \varphi}$. So from $(G 1),(G 2),(G 3)$ we will have only $2 n$ independent equations in the $3 n$ components (of degree 2 in the $x_{i}$ ) of $A, B$, and $C$. (Moreover this proves that if $b \neq 0$, we will always have exactly $2 n$ independant equations in the $(\simeq 3 n)$ components of $A, B$ and $C$ ).

\section{Implementations and public key lengths}

The algorithms Dragon and MIIP-3 that we have seen are very efficient. These algorithms are fast and can easilly be implemented in smartcards with low power (without arithmetic coproccssor). Moreover we will see now that the public key length can be very moderate for two reasons:

1. We can have a value $n$ which is not too large (for example $n=32$ ) if wc have a value $q$ which is not too small.

2. Moreover, the public key can be written with polynomials of total degree two (instead of threc) as we will see now! (Unfortunately this idea will help us to attack the schemes as we will see in Part 2).

\section{Dragon}

In the Dragon algorithms of paragraph 2, the hidden equation is:

$$
a^{q^{\theta}+q^{\varphi}} \cdot M(b)=a^{q^{\zeta}+q^{\xi}} \cdot N(b) .
$$

For simplicity of the equations lot us assume that $s$ and $t$ are linear (if $s$ and $t$ are affine the same results will also hold). The public key, computed from this equation (6) is a set of $n$ equations like this:

$$
\sum \gamma_{i j k l} x_{j} x_{k} y_{l}=0 . \quad ;=1 \ldots, n
$$


Let $P_{i l}=\sum_{j, k} \gamma_{i j k l} x_{j} x_{k}$. We have $O\left(n^{2}\right)$ such values $P_{i j}$. But how many independant such $P_{i l}$ will we have? In fact at most $2 n$ because in the hidden equations (6) we have $n$ components from $a^{q^{\mu}+q^{\varphi}}$ and $n$ components from $a^{q^{\zeta}+q^{\xi}}$. So all the $P_{i l}$ can be written as a linear expression in only $\lambda$ variables, $\lambda \leq 2 n$, that we will call $p_{1}, \ldots, p_{\lambda}$. So we see that the public key can always be written (without changing the security since this new public key can be computed from the original in polynomial complexity) as two sets of equations.:

$$
\begin{gathered}
p_{i}=\sum_{j, k} \nu_{i, j} x_{j} x_{k} \quad(i=1 \text { to } \lambda, \quad \lambda \leq 2 n) \\
\sum_{j=1}^{\lambda} \sum_{k=1}^{n} \xi_{i j k} p_{j} y_{k}=0 \quad(i=1 \text { to } n) .
\end{gathered}
$$

In these new public equations we will have only $0\left(n^{3}\right)$ terms instead of $0\left(n^{4}\right)$ terms in the original presentation.

Note. If $a^{q^{\theta}+q^{\varphi}}$ is not a linear transformation of $a^{q^{\zeta}+q^{\xi}}$ then $\lambda=2 n$ with a very high probability. It is not clear if $\lambda$ can be $\neq n, \simeq 2 n$ and $<2 n$ and what cryptanalysis can be done if this occur

\section{MIIP-3}

Similarly, from the public key of a MIIP-3 algorithm it is always feasable to compute all the equations $\sum \gamma_{i j k} x_{i} x_{j} y_{k}+\cdots=0$ that are always true when $x$ is the encryption of $y$, and to see that the terms in the $y_{k}$ variables are generated by only about $0(n)$ polynomials of dcgree two. 'The fact that we always have such polynomials come from the (G1), (G2), (G3) cquations. If we denote by $P_{1}, \ldots, P_{\lambda}$ these polynomials $(\lambda=0(n)$ and $\lambda=3 n$ very often $)$, then instead of the public key we can write (without changing the security) about $\mu$ equations like this, $\mu \simeq 3 n$ :

$$
\sum_{k=1}^{n} \sum_{j=1}^{\lambda} \xi_{i j k} p_{j} y_{k}+\sum \mu_{i j} p_{j}+\sum \nu_{i j} y_{j}+\delta_{i}=0, \quad 1 \leq i \leq \mu .
$$

(Most of these equations come from (G1), (G2), (G3). However sometimes we will have $\mu \simeq 4 n$ or $\mu \simeq 5 n$ when we will have more equations than (G1), (G2), (G3). But $\mu$ is always such that $\mu \simeq k n$, with $k$ small and $k \geq 3$ ).

These equations plus the definitions of $p_{1}, \ldots, p_{\lambda}$ are the new public key and we have about $0\left(n^{3}\right)$ terms in the new public key instead of $0\left(n^{4}\right)$ terms in the original public key.

Note. The designer of the scheme can choose to make public only a part of these equations, for example these coming from (G1). This may make the attack 
easier (as we will see in Part 2) since he has isolate (G1) from (G2) and (G3). However since anybody can compute the set of all the equations as above it does not change de security, from the polynomial complexity point of view, to present this set of $0\left(n^{3}\right)$ equation as the new public kcy.

\section{PART 2: Cryptanalysis results}

\section{Cryptanalysis of extended Matsumoto-Imai Al- gorithms with small branches}

In the description of the original Matsumoto-Imai $C^{*}$ algorithm given in [1], after the first affine transformation $s$, the inputs are divided in $d$ branches. We have not made such a separation in our description of Dragon and MIJP-3, because we have found three very general attacks against small branches. We describe these three attacks in the cxtended version of this paper. These threc attacks are very instructive and they are based on three completely different ideas. The first one uses some algebraic equations, and the second one is based on differential cryptanalysis.

\section{Cryptanalysis of two compositions of $C^{*}$ algo- rithms}

A very natural idea, in order to keep a bijective cryptosystem with easy secret computations is to do the composition of two $C^{*}$ Matsumoto-Imai Algorithms. Of course one problem is that the public polynomials will be of total degree four (instead of two) but if $n$ is not too big, so if $K=\mathbb{F}_{q}$ is not so small $\left(q=2^{\mu}\right.$, with $\mu \neq 1$ ), then the length of the public key may still bc acceptable. However we show in the extended version of this paper a bigger problem: such a scheme remains insure.

We will just give here the idea of the attack. It is to compute all the equation of this form:

$\sum_{i} \sum_{j} \sum_{k} \mu_{i j k} y_{i} x_{j} x_{k}+\sum_{i} \sum_{j} \nu_{i j} x_{i} x_{j}+\sum_{i} \sum_{j} \xi_{i j} y_{i} x_{j}+\sum_{i} w_{i} x_{i}+\sum_{i} \theta_{i} y_{i}+\pi_{0}=0$.

Then we will introduce some "transition" variables $p_{i}$ such that these equationscan bc written like this:

$$
\sum_{i} \sum_{j} \gamma_{i j}^{\prime} p_{i} y_{j}+\sum_{i} \alpha_{i}^{\prime} p_{i}+\sum_{i} \beta_{i}^{\prime} y_{i}+\delta_{0}^{\prime}=0
$$

when the $y_{i}$ variables are given, then from these equations we will be able to find the $p_{j}$ variables (by Gaussian reduction). Then we will find $x$ from the $p_{j}$ variables as in the altack of [2]. 


\section{Cryptanalysis of the little Dragon Algorithm}

\section{Introduction}

In this paragraph we will study the cryptanalysis of the "little Dragon" algorithm. What we call "little Dragon algorithm" is the algorithm where instead of equation (1) (of paragraph 2.2) the hidden equation is:

$$
a \cdot b=a^{q^{\theta}+q^{\varphi}}
$$

where $\theta$ and $\varphi$ are secret integers such that $h=q^{\theta}+q^{\varphi}-1$ is coprime with $q^{n}-1$. Since there are not a lot of possible values for $\theta$ and $\varphi$ we can also assume that $\theta$ and $\varphi$ are public.

This algorithm looks very interesting because the public equations computed from (7) are only of total degree two. However this algorithm is insecure, as we will see now.

\section{Cryptanalysis of the scheme}

We will assume here that the secret functions $s$ and $t$ are linear (not only affine). This probably does not change a lot of things (moreover there the value $a=0$ can very easily be detected so we can clearly assume that $s$ is linear). So the public equations comming from (7) is a set of $n$ equations like this:

$$
\sum_{j, k} \gamma_{i j k} x_{j} y_{k}+\sum_{j, k} \mu_{i j k} x_{j} x_{k}=0, \quad 1 \leq i \leq n
$$

Let $\delta_{i}=\sum \gamma_{i j k} x_{j} y_{k}, 1 \leq i \leq n$. The values $\delta_{i}$ are public and they represent the "hidden" components of $a \cdot b$. We denote by $\delta=\left(\delta_{1}, \ldots, \delta_{n}\right)$.

The cryptanalysis is in four steps.

Step 1 We compute the vector space of all the linear transformations $C$ and $D$ such that:

$$
\forall i, \quad 1 \leq i \leq n, \quad(C(\delta))_{i}=\sum_{j, k} \gamma_{i j k} x_{j}(D(y))_{k} .
$$

This set will by found by Gaussian reductions on the values of the $C$ and $D$ matrices.

We are sure to find a vector space of solutions of dimension at least $n$ since we have :

$$
\forall \lambda \in F_{q^{n}}, \quad \lambda(a \cdot b)=a \cdot(\lambda b) .
$$

So each transformation $b \mapsto \lambda b$ gives a couple $(C, D)$ of matrices solution.

Moreover I did a small simulation and in this simulation I found a dimension exactly $n$ for the set of solutions. For simplicity, let us assume that we have exactly such a dimension $n$ of solutions.

Since the set of solutions found for $D$ depends on $n$ free variables, we can call these variables $\Lambda_{1}, \Lambda_{2}, \ldots, \Lambda_{n}$, and we can denote $\Lambda=\left(\Lambda_{1}, \ldots, \Lambda_{n}\right)$, and 
$D_{\Lambda}$ the solution with the parameter $\Lambda$. We will also denote by $D_{\Lambda}(y)$ the vector of components $\left(D_{\Lambda}\left(y_{1}\right), \ldots, D_{\Lambda}\left(y_{n}\right)\right)$.

Step 2 We compute the vector space of all the linear transformations $E$ such that :

$$
D_{E(\Lambda)}(y)=D_{E(y)}(\Lambda) .
$$

Here again we expect to find a vector space of dimension $n$ (and indeed this is what I found in my small simulation). Let $E_{0}$ be such a solution and let $*$ be the operation such that by definition:

$$
\Lambda * y=y * \Lambda=D_{E_{0}(\Lambda)}(y) .
$$

Remark If we denote by $t$ the sccret affinc transformation from $b$ to $y$, then there will be an element $\mu \in F_{q^{n}}, \mu \neq 0$, such that:

$$
\Lambda * y=t\left(\mu \cdot t^{-1}(\Lambda) \cdot t^{-1}(y)\right)
$$

So $t$ and $\mu$ are not known, but such an operation $*$ has been found.

Step 3 Let $h=1+q^{\theta}+q^{\varphi}$, so $b=a^{h}$, and let $h^{\prime}$ be the inverse of $h$ mod $2^{n}-1$, so $a=b^{h^{\prime}}$. We can assume that $h^{\prime}$ is public because there are not a lot of possible values for $\theta$ and $\varphi$ (so the cryptanalyst can try one by one all the solutions).

Let $f$ be the function : $f(y)=y^{h^{\prime}}$, where $y^{h^{\prime}}$ denotes $y * y \cdots * y, h^{\prime}$ times. (This function $f$ is computed by square and multiply from the operation $*$ ). Then we have: $f(y)=t\left(\mu^{h^{\prime}-1} b^{h^{\prime}}\right)=t\left(\mu^{h^{\prime}-1} a\right)$ (where $b$ denotes $t^{-1}(y)$ as usual). So $f(y)=W(x)$, where $x$ is the cleartext and $W$ an affine function! So from $f$ it is easy to find $W$ by Gaussian reductions on a few cleartext/ciphertext couples.

Step 4 Now that $f$ and $W$ are found it is easy to decrypt any message since: $x=W^{-1}(f(y))$.

\section{Cryptanalysis of the Dragons of paragraph 2}

Now we will give an algorithm for the cryptanalysis of the Dragon scheme given in paragraph 2.

For simplicity we will assume that $M$ (or $N$ ) is bijective and that $s$ and $t$ are linear. (This probably does not change much). So we can assume that the hidden equation is:

$$
a^{q^{\theta}+q^{\varphi}} \cdot b=a^{q^{\zeta}+q^{\xi}} \cdot N(b) .
$$

Since $s$ and $t$ are linear we know from paragraph 4 that the public key can be given as a set of about $2 n$ equations like this: $p_{i}=\sum_{j=1}^{n} \sum_{k=1}^{n} \nu_{i j k} x_{j} x_{k}, 1 \leq i \leq 2 n$, 
plus a set of $n$ equations like this: $\sum_{j=1}^{2 n} \sum_{k=1}^{n} \xi_{i j k} p_{j} y_{k}, 1 \leq i \leq n$. (From the public key it is always feasable to find these two sets of equations).

Let $\delta_{i}=\sum_{j=1}^{2 n} \sum_{k=1}^{n} \xi_{i j k} p_{j} y_{k}$. The values $\delta_{i}, 1 \leq i \leq n$ are public. We denote by $\delta=\left(\delta_{1}, \ldots, \delta_{n}\right)$, and by $p=\left(p_{1}, \ldots, p_{2 n}\right)$. The cryptanalysis is in four steps.

Step 1 We compute (by Gaussian reductions) the vector space of all the linear transformations $C$ and $D$ such that:

$$
\forall i, \quad 1 \leq i \leq n, \quad(C(\delta))_{i}=\sum_{j=k}^{2 n} \sum_{k=1}^{n} \xi_{i, j k}(D(p))_{j} y_{k}
$$

$C$ is a $n \times n$ matrix and $D$ is a $2 n \times 2 n$ matrix.

We are sure to find a vector space of solutions of dimension at least $n$ since we have :

$$
\forall \lambda \in F_{q^{n}}, \quad \lambda\left(a^{q^{\theta}+q^{\varphi}} \cdot b-a^{q^{\zeta}+q^{\xi}} \cdot N(b)\right)=\left(\lambda a^{q^{\theta}+q^{\varphi}}\right) \cdot b-\left(\lambda a^{q^{\zeta}+q^{\xi}}\right) \cdot N(b) .
$$

So each transformation $\left(a^{q^{\theta}+q^{\varphi}}, a^{q^{\zeta}+q^{\xi}}\right) \mapsto\left(\lambda a^{q^{\theta}+q^{\varphi}}, \lambda a^{q^{\zeta}+q^{\xi}}\right)$ gives a couple $(C, D)$ of matrices solution.

Step 2 Let $D_{0}$ be such a solution, with $D_{0} \neq 0$. Then we will find an invertible matrix $S$ such that $D_{0}=S^{-1} D_{0}^{t} S$ where

$$
D_{0}^{\prime}=\left(\begin{array}{ccc}
D_{1} & \vdots & 0 \\
\cdots & \vdots & \ldots \\
0 & \vdots & D_{2}
\end{array}\right)
$$

where $D_{1}$ and $D_{2}$ are two $n \times u$ matrices. (This is feasable from the matrices reduction theory, however I did no simulation. Sec for example [4]). $D_{1}$ will come from $a^{q^{\theta}+q^{\varphi}} \mapsto \lambda a^{q^{\theta}+q^{\varphi}}$ and $I_{2}$ will come from $a^{q^{\zeta}+q^{\epsilon}} \mapsto \lambda a^{q^{\zeta}+q^{\xi}}$.

Step 3 The matrix $S$ gives a change of variables on the $p_{i}$ variables: let $p_{1}^{\prime}, \ldots, p_{n}^{\prime}$ be the terms changed by $D_{1}$ and $q_{1}^{\prime}, \ldots, q_{n}^{\prime}$ be the terms changed by $D_{2}$. Then the $n$ equations $\delta_{i}$ can be rewritten in $n$ equations like this:

$$
\sum_{j=1}^{n} \sum_{k=1}^{n} \mu_{i j k} y_{j} p_{k}^{\prime}=\sum_{j=1}^{n} \sum_{k=1}^{n} \gamma_{i j k} y_{j} q_{k}^{\prime}, \quad 1 \leq i \leq n
$$

Step 4 Let $\delta_{1}^{\prime}, \ldots, \delta_{n}^{\prime}$ be the terms of the left side of this equation:

$$
\delta_{i}^{\prime}=\sum_{j=1}^{n} \sum_{k=1}^{n} \mu_{i, j k} y_{j} p_{k}^{\prime}, \quad 1 \leq i \leq n
$$


These terms come from $a^{q^{\theta}+q^{\psi}} \cdot b$. From these terms we will find an operation $\Lambda * y$ exactly as we did for the little Dragon algorithm. So if $a=b^{h^{\prime}}$ (as in paragraph 2 example 2 with $\nu=0$ or as in the (G1) equation of MIIP-3) then we will just compute $y^{h^{\prime}}$ with this * operation. What about more general cases, i.e. when the transformation from $b$ to $a$ is more complex than $b=a^{h^{\prime}}$ ? Duc to the lack of space please see the extended version of this paper. (The idea is to find the analogy of $b \mapsto(N(b) / b)^{h^{\prime}}$ in $y$ with the * operation as the basic operation on $y$ ).

\section{Cryptanalysis of MIIP-3}

Now we will give an algorithm for the cryptanalysis of the MIIP-3 algorithm. For simplicity we will assume that $s$ and $t$ are linear (this probably docs not change a lot of things). Since $s$ and $t$ are linear we know from paragraph 4 that the public key can be given as a set of about $3 n$ equations like this (sometimes more): $p_{i}=\sum_{j=1}^{n} \sum_{k=1}^{n} v_{i j k} x_{j} x_{k}, 1 \leq i \leq 3 n$, plus a set of about $3 n$ equations like this: $\sum_{j=1}^{3 n} \sum_{k=1}^{n} \xi_{i j k} p_{j} y_{k}=0,1 \leq i \leq 3 n$. These equations come from

$$
(G 1): B \cdot b^{q^{\theta}}=b \cdot C^{q^{\varphi}},(G 2): A \cdot b^{q^{\varphi}}=b \cdot C^{q^{\varphi}},(G 3): A^{q^{\theta}} \cdot b^{q^{\varphi}}=b^{q^{\theta}} \cdot B^{q^{\varphi}}
$$

where $A=a^{1+q^{*}}, B=a^{1+q^{\varphi}}$, and $C=a^{q^{\theta}+q^{\varphi}}$. (Sometimes we have more than these $3 n$ equations, but for simplicity we will assume that there are only these $3 n$ equations and that they give a vector space of dimension $3 n$ ).

Let $\delta_{i}=\sum_{j=1}^{3 n} \sum_{k=1}^{n} \xi_{i j k} p_{j} y_{k}$. The values $\delta_{i}, 1 \leq i \leq 3 n$, are public. We denote by $\delta=\left(\delta_{1}, \ldots, \delta_{3 n}\right)$ and by $p=\left(p_{1}, \ldots, p_{3 n}\right)$. The cryptanalysis is in three steps.

Step 1 We computc (by Gaussian reductions) the vector space of all the lincar transformations $D$ and $E$ such that:

$$
\forall i, \quad 1 \leq i \leq n, \quad(E(\delta))_{i}=\sum_{j=k}^{3 n} \sum_{k=1}^{n} \xi_{i j k}(D(p))_{j} y_{k}
$$

$E$ is a $3 n \times 3 n$ matrix and $D$ is also a $3 n \times 3 n$ matrix.

We are sure to find a vector space of solutions of dimension at least $n$ since we have : $\forall \lambda \in F_{q^{n}}$, if $B$ is changed in $\lambda^{q^{\theta}} B, G$ in $\lambda C$ and $A$ in $\lambda^{q^{\varphi}} A$, then $(G 1)$ is changed in $\lambda^{q^{\theta}}(G 1),(G 2)$ in $\lambda^{q^{\phi}}(G 2)$ and $(G 3)$ in $\lambda^{q^{\theta}+q^{\varphi}}(G 3)$.

Step 2 Let $E_{0}$ be such a solution for $E$, with $E_{0} \neq 0$. 'Then we will find an invertible matrix $S$ such that $E_{0}=S^{-1} E_{0}^{\prime} S$ where 


$$
E_{0}^{\prime}=\left(\begin{array}{ccccc}
E_{1} & \vdots & 0 & \vdots & 0 \\
\cdots & \vdots & \ldots & \vdots & \ldots \\
0 & \vdots & E_{2} & \vdots & 0 \\
\cdots & \vdots & \cdots & \vdots & \cdots \\
0 & \vdots & 0 & \vdots & E_{3}
\end{array}\right)
$$

(This is feasable from the matrices reduction theory. See for example [4]).

$E_{1}$ comes from $(G 1) \rightarrow \lambda^{q}(G 1), E_{2}$ comes from $(G 2) \rightarrow \lambda^{q}(G 2)$ and $E_{3}$ comes from $(G 3) \rightarrow \lambda^{q^{\theta}+q^{\varphi}}(G 3)$.

Step 3 From $S$ we can isolate the equation $(G 1)$ from the other equations, and so attack the scheme as a Dragon scheme as we did in paragraph 8 .

Remark. For MIIP-4 (i.e. with the hidden equation $b=a^{1+q^{\theta_{1}}+q^{\theta_{2}}+q^{\theta_{3}}}$ ) the same kind of polynomial attack exists.

\section{Unclear cases}

In all the schemes. Is the cryptanalysis more difficult if the transformations $s$ and $t$ are affine (instead of linear)? (Probably not, but I did not check).

For MIIP-3 and $C^{*}$. In MIIP-3, with the original public form, or in $C^{*}$, what do we do if 2 or 3 of the public polyomials are not given? The scheme will still work in signature, and also in encryption if we had redundancy, but may be more difficult to attack. (However if only one equation is not given with $n=64$ and $K=F_{2}$ then from the Birthday paradox we will easilly be able to find this equation).

\section{PART 3: A candidate for 64 bits signatures and conclusion}

\section{A candidate Dragon algorithm for extremely short signatures}

$\forall a \in F_{q^{n}}, \forall b \in\left(F_{q}\right)^{2 n}$, let

$$
f(a, b)=\sum_{i=1}^{k} \alpha_{i} a^{q^{\beta_{i}}+q^{\gamma_{i}}} N_{i}(b)+\sum_{i=1}^{k^{\prime}} \alpha_{i}^{\prime} a^{q^{\beta_{i}^{\prime}}} N_{i}^{\prime}(b)+\delta_{0}
$$

where for all the indices $i$ we have: $\alpha_{i}, \alpha_{i}^{\prime}, \delta_{0} \in F_{q^{n}}, \beta_{i}, \gamma_{i}, \beta_{i}^{\prime}, k$ and $k^{\prime}$ are integers, $N_{i}$ and $N_{i}^{\prime}$ are affine functions of $\left(F_{q}\right)^{2 n} \rightarrow F_{q^{n}}$ (as usual affine means 
affine over $F_{q}$ ), and where the degree $d$ in the a variable is not too large (for example $d \leq 8000$ ).

Then let $a=s(x)$ and $b=t(y)$ where $s$ and $t$ are two secret affine permutations. In a basis $f(a, b)=0$ gives $n$ cquations of total degree three (degree two in $a_{i}$ and one in $b_{i}$ variables). These $n$ equations will be writen in $x_{i}$ and $y_{j}$ variables (Step 1) and then a linear and bijective transformation is done on these equations (Step 2). We obtain like this a set $(G)$ of $n$ equations of total degree three (degree two in $x_{i}$ and one in $y_{i}$ variables). $(G)$ is public. $f$ is secret (this function is "hidden" by $s$ and $t$ ).

For example $q=2, n=64$, and in $f$ we have all the monomials $a^{2^{\beta_{i}}+2^{\gamma_{i}}}$ and all the monomials $a^{2^{\beta_{i}^{\prime}}}$ with $2^{\beta_{i}}+2^{\gamma_{i}}<8000$ and $2^{\gamma_{i}}<8000$, and $\alpha_{i}$ and $\alpha_{i}^{\prime}$ are randomly chosen in $F_{q^{n}}$, and $N_{i}$ and $N_{i}^{\prime}$ are also randomly chosen.

Let $M$ be a message to sign. The signature of $M$ is $(x \| R)$ where $R$ is any small integer with no pattern 1000 in its expression in base 2 (for example $R=0$ ). $x$ is any 64 bits value such that if we denote $y=\operatorname{Hash}(R\|1000\| M)$, then $(x, y)$ satisfies all the $n$ equations of $(G)$. Here Hash is a collision free Hash function with 128 bits outputs (for example Hash $=M D 5$ ), and $\|$ is the concatenation function. So anybody can verify a signature $(x, R)$ without any secret. In order to compute the signature we will compute $b=l^{-1}(y)$, then we will solve in $a$ the equation $f(a, b)=0$ (this is always feasable with a complexity polynomial in $d$ ). If there is no solution, we try with another value $R$ (for example $R=1$ instead of $R=0$ ) until we find a solution $a$. Then $x=s^{-1}(a)$ is computed. On average the length of the signature $(R, x)$ will only be about 64 bits. (Moreover we can also give only $x$ as the signature and all the small values of $R$ will be tried one by one to check the signature). We avoid the "birthday paradox" since we can not publicly compute $y$ from $x$ but just check if $x$ and $y$ match together or not. However the time to compute a signature is long so this schemc is not very efficient. Its interest lies in the fact that it is the first candidate algorithm with 64 bits asymmetric signatures (I do not know any previous candidate). The best attack that $I$ know against this scheme needs more lhan $2^{50}$ computations. With 80 bits signature this attack needs more than $2^{64}$ computations. Moreover after these computations, only one signature is found: to compute another signature the same huge computations are needed.

This algorithm is still a Dragon algorithm (since $x$ and $y$ are mixed) but with a hidden function instead of a hidden monomial.

Note 1 These attacks are based on the idea to do cxhaustive search on $n-k$ variables $x_{i}, k$ small, and to find the $k$ other variables from the public equations. I was no able to see somebody who knows if a better attack is known to solve a randomly set on $n$ quadratic equations over $G F(2)$ when $n \simeq 64$. I will try to have the efficiency of the known algorithms for the conference in August. Maybe $n=64$ is easy even for random quadratic equations?

Note 2 For any signature scheme with signatures of length 64 bits that can sign messages of arbitrary length, after about $2^{32}$ signatures two messages signed have the same signaturc. However here the collision is obtained between two messages 
signed by the owner of the secrets, and moreover only after $2^{32}$ signatures, made by himself. So this may not be a problem.

Note 3 The function $f$ (as in a variation of HFE) can also be more general, as long as in a basis $f(a, b)$ is of small degree in $a_{i}$ and $b_{i}$ variables and the computation of a such that $f(a, b)=0$ is feasable. For example a multivariate resolution algorithm with a few variables ( 8 equations with 8 variables for example with each variable on 8 bits) will be hidden as an intractable system of 64 equations with 64 variables $a_{i}$ and 64 variables $b_{i}$ by $s$ and $t$.

\section{Conclusion}

In this paper, we have studied some algorithms based on the idea of a "hidden" monomial. The motivation was that these algorithms are very efficient and that some of these algorithms were candidate trapdoor one way permutations. Unfortunately we have seen that all the easy transformations of $C^{*}$ can be attacked in polynomial complexity. (Some simulations would be require to test the validity of the attacks). We have also described a candidate algorithm for 64 bits signatures that has so far resisted all attacks. However this algorithm is not very efficient and also has no proof of security. So at present, the two algorithms of [3] seem to remain the best candidates to try to repair the $C^{*}$ algorithm of [1].

\section{Acknowledgments}

I want to thank Isabelle Guerrin-Lassous for doing a lot of simulations, Henri Gilbert for his help in the design of the differential attack of paragraph 5; also Frédéric Charbonier, 'Isutomo Matsumoto, Louis Gaubin and Paul Camion for useful comments. Finally I would like to give special thanks to an anonymous referee of Crypto' 96 for giving the idea to study the transformation $b \mapsto \lambda b$ on the public equations of the little Dragon algorithm...

\section{References}

[1] T. Matsumoto and H. Imai, "Public quadratic polynomial-tuples for efficient signature-verification and message-encryption", EUROCRYPT'88, Springer Verlag, pp. 419-453.

[2] J. Patarin, "Cryptanalysis of the Matsumoto and Imai public key scheme of Eurocrypt'88", CRY P'TO'95, Springer Verlag, pp. 248-261.

[3] J. Patarin, "Hidden Fields Equations ( $\mathrm{HF}^{3} \mathrm{E}$ ) and Isomorphisms of Polynomials (IP): two new Families of Asymmetric Algorithms", EUROCRYPT'96, Springer Verlag, pp. 33-48.

[4] F.R. Gantmacher, "The theory of matrices", Chelsea Publishing Company, Volume one, chapter VII. 